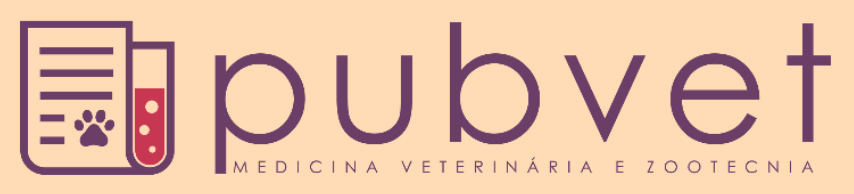

https://doi.org/10.31533/pubvet.v14n4a549.1-6

\title{
Genotoxicidade em Danio rerio expostos a concentrações crescentes da fração solúvel do biodiesel
}

\author{
Dênio Garcia de Oliveira ${ }^{1}$, Daniella Aparecida de Jesus Paula ${ }^{2} \bullet$, Luis David Solis Murgas ${ }^{3 *} \bullet$ \\ ${ }^{1}$ Professor do Centro Universitário de Formiga, Instituto de Ciências da Saúde. Formiga-MG. Brasil. \\ ${ }^{2}$ Pós-doutoranda na Universidade Federal de Lavras, Programa de Pós-graduação em Ciências Veterinárias. Lavras-MG. Brasil. \\ ${ }^{3}$ Professor da Universidade Federal de Lavras, Departamento de Medicina Veterinária. Lavras-MG. Brasil. \\ *Autor para correspondência, E-mail: lsmurgas@ufla.br
}

Resumo. Objetivou-se, com esta pesquisa, determinar a presença de micronúcleos em eritrócitos de peixes juvenis de zebrafish (Danio rerio), expostos a concentrações crescentes de fração solúvel do biodiesel (FSB) de óleo de soja queimado. Os peixes $(\mathrm{N}=15)$ foram alocados em aquários de polietileno e submetidos ao teste de toxicidade aguda e um delineamento em bloco inteiramente casualizado, com seis tratamentos e duas repetições. Os tratamentos foram diferentes concentrações de FSB, sendo as soluções: I (controle), II $\left(0,0645 \mathrm{~g} \mathrm{~L}^{-1}\right)$, III $\left(0,129 \mathrm{~g} \mathrm{~L}^{-1}\right), \mathrm{IV}\left(0,645 \mathrm{~g} \mathrm{~L}^{-1}\right), \mathrm{V}\left(1,29 \mathrm{~g} \mathrm{~L}^{-1}\right)$ e VI $(6,45 \mathrm{~g}$ $\left.\mathrm{L}^{-1}\right)$. Foi realizado um esfregaço sanguíneo para cada peixe, sendo analisados 2000 eritrócitos intactos. Houve diferença significativa $(\mathrm{p}<0,05)$ entre o grupo I, II, III e IV e os grupos V e VI, nos tempos 24 e 96 horas de exposição. Diante dos resultados, podemos observar que a FSB tem efeito genotóxico em eritrócitos de peixes juvenis de zebrafish.

Palavras chave: biocombustível, eritrócitos, teste do micronúcleo, toxicidade, zebrafish

\section{Genotoxicity in Danio rerio exposed to increasing concentrations of soluble fractioning of burnt soybean oil biodiesel}

\begin{abstract}
The objective of this research, to determine the presence of micronuclei in erythrocytes of juvenile fish zebrafish (Danio rerio) exposed to increasing soluble fraction concentrations of burnt soybean oil biodiesel (FSB). The fishes $(\mathrm{N}=15)$ were placed in polyethylene aquariums, with constant aeration, and submitted to acute toxicity test in a completely randomized design, with six treatments and two replicates. The treatments consisted of different FSB concentrations: solution I (control), solution II $\left(0.0645 \mathrm{~g} \mathrm{~L}^{-1}\right)$, solution III $\left(0.129 \mathrm{~g} \mathrm{~L}^{-1}\right)$, solution IV $\left(0.645 \mathrm{~g} \mathrm{~L}^{-1}\right)$, solution V $\left(1.29 \mathrm{~g} \mathrm{~L}^{-1}\right)$ and solution VI (6.45 $\left.\mathrm{g} \mathrm{L}^{-1}\right)$. We collected a blood smear lamina for each fish, analyzing 2000 intact erythrocytes. There was significant difference $(\mathrm{p}<0.05)$ between groups I (control), II, III and IV and groups V and VI in the periods of 24 and 96 hours of exposition. With these results we may observe that the FSB presents a genotoxic effect in the erythrocytes of juvenile fish zebrafish.
\end{abstract}

Keywords: micronucleus test, zebrafish, erythrocytes, biofuel, toxicity

\section{Genotoxicidad en Danio rerio expuestos a concentraciones crecientes de la fracción soluble del biodiesel de aceite de soja quemado}

Resumen. El objetivo de esta investigación es determinar la presencia de micronúcleos en los eritrocitos de pez cebra (Danio rerio) expuestos a concentraciones crecientes de 
fracción soluble del biodiesel de aceite de soja quemado (FSB). Los peces $(\mathrm{N}=15)$ se colocaron en acuarios de polietileno, con aireación constante, y se sometieron a una prueba de toxicidad aguda en un diseño completamente al azar, con seis tratamientos y dos repeticiones. Los tratamientos consistieron en diferentes concentraciones de FSB: solución I (control), solución II $\left(0.0645 \mathrm{~g} \mathrm{~L}^{-1}\right)$, solución III $\left(0.129 \mathrm{~g} \mathrm{~L}^{-1}\right)$, solución IV $\left(0.645 \mathrm{~g} \mathrm{~L}^{-1}\right)$, solución V (1.29 $\left.\mathrm{g} \mathrm{L}^{-1}\right)$ y solución VI $\left(6,45 \mathrm{~g} \mathrm{~L}^{-1}\right)$. Fue elaborado un frotis de sangre para cada pez, analizando 2000 eritrocitos intactos. Hubo una diferencia significativa $(p<0.05)$ entre los grupos I (control), II, III y IV y los grupos V y VI en los períodos de exposición de 24 y 96 horas de exposición. Con estos resultados podemos observar que el FSB presenta un efecto genotóxico en los eritrocitos de peces cebra jóvenes.

Palabras clave: prueba de micronúcleos, pez cebra, eritrocitos, biocombustible, toxicidad

\section{Introdução}

Uma maneira de verificar os efeitos tóxicos em uma população aquática exposta a agentes químicos e/ou biológicos é conduzir estudos de monitoramento, utilizando-se parâmetros biológicos, de preferência, estudos com manifestações em curto prazo, tais como ensaios de toxicidade aguda. Para avaliar a ocorrência de prováveis mutações ou alterações genéticas em uma célula é preciso utilizar algumas técnicas, dentre elas o Teste de Micronúcleos. Esse consiste na identificação do aumento na frequência de mutação em células, que são expostas a uma gama variada de agentes genotóxicos. $O$ teste alia simplicidade, análise rápida de um elevado número de células, menor custo, grande poder estatístico, velocidade e facilidade de análise, utilização de pequenas concentrações de células, além da eficácia em danos clastogênicos e aneugênicos (Santos, 2010; Swanson et al., 2007).

O micronúcleo é um núcleo adicional e separado do núcleo principal de uma célula, formado na telófase, durante a divisão celular por cromossomos ou fragmentos de cromossomos que se atrasam em relação aos demais. O teste do micronúcleo pode ser aplicado em qualquer tecido animal, sendo dependente da proporção de células que estão se dividindo, ocorrendo, durante um tempo equivalente ao ciclo celular, que resulta de alterações estruturais cromossômicas espontâneas ou experimentalmente induzidas ou de falhas no fuso celular (Fenech, 1997; Simonato et al., 2006).

Diante disso, objetivou-se determinar a presença de micronúcleos em eritrócitos de zebrafish, expostos a concentrações crescentes de fração solúvel do biodiesel de óleo de soja queimado.

\section{Material e métodos}

\section{Animais}

Nesta pesquisa, exemplares $(\mathrm{n}=15)$ juvenis de zebrafish (Danio rerio), pesando, aproximadamente $0,89 \pm 0,177 \mathrm{~g}$, adquiridos de Loja de Aquarismo foram aclimatados durante aproximadamente 48 horas, em aquário de polietileno de $50 \mathrm{~L}$, no laboratório de Fisiopatologia da Reprodução, Parasitologia e Análises Clínicas do Centro Universitário de Formiga/MG. Durante o período experimental, os peixes foram alimentados ad libitum com ração comercial, duas vezes ao dia.

\section{Obtenção do biodiesel e preparo da fração solúvel do biodiesel (FSB)}

O biodiesel foi adquirido no Laboratório de Biocombustível da Universidade Federal de Lavras, sendo obtido por metilação do óleo de soja utilizado em frituras, portanto não estava padronizado e nem purificado.

A metodologia para separação da fração solúvel do biodiesel (FSB) foi modificada de $\underline{\text { Simonato et }}$ al. (2006). Misturou-se uma parte de biodiesel em quatro partes de água que foi levada ao agitador magnético, durante 45 minutos. Após esse, tempo colocou-se a solução no decantador durante 60 minutos e retirou-se a fração solúvel, descartando o sobrenadante. Essa preparação foi feita no Laboratório de Química do Centro Universitário de Formiga. Foi encontrada a concentração do biodiesel na solução: $0,0645 \mathrm{~g} \mathrm{~L}^{-1} \mathrm{e}$ sua composição consta na Tabela 1. 
Tabela 1. Resultados analíticos do biodiesel

\begin{tabular}{|c|c|c|c|}
\hline Parâmetro & Unidade & Resultado & Metodologia \\
\hline Etanol & $\% \mathrm{v} \mathrm{v}^{-1}$ & Não detectado & $\mathrm{CG}$ \\
\hline Hidróxido de Sódio & $\mathrm{Mg} \mathrm{L}^{-1}$ & Não detectado & Titulométrico \\
\hline Lipídeos & $\mathrm{g} 100 \mathrm{~mL}^{-1}$ & 0,003 & IAL 032-34/IV \\
\hline Metanol & $\% \mathrm{v} \mathrm{v}^{-1}$ & 0,24 & POP. CROMO.14 \\
\hline
\end{tabular}

Fonte: HIDROCEPE Laudo de Análise no 12571 (04/02/2013).

\section{Teste de toxicidade}

Os peixes foram submetidos ao teste de toxicidade aguda, no qual são coletadas amostras com 6, 24 e 96 horas após exposição ao agente, em aquários de polietileno, com capacidade para três litros de água, com aeração constante, em um delineamento em bloco inteiramente casualizado (DBC), sendo seis tratamentos com duas repetições, totalizando doze aquários. Em cada unidade experimental, contendo diferentes concentrações de FSB, foram colocados 15 peixes, totalizando 180 animais e as concentrações definidas com base no trabalho de Masouleh et al. (2011), conforme Tabela 2.

Tabela 2. Concentrações da FSB utilizadas no experimento.

\begin{tabular}{lcc}
\hline Tratamento & Concentração de biodiesel g L-1 & Fator de diluição \\
\hline Solução I & Água do tanque (controle) & 0 \\
Solução II & 0,0645 & $1.1000^{-1}$ \\
Solução III & 0,129 & $1.500^{-1}$ \\
Solução IV & 0,645 & $1.100^{-1}$ \\
Solução V & 1,29 & $1.50^{-1}$ \\
Solução VI & 6,45 & $1.10^{-1}$ \\
\hline
\end{tabular}

\section{Teste do micronúcleo}

O teste do micronúcleo foi realizado de acordo com metodologia descrita por Al-Sabti \& Metcalfe (1995) e a coleta de sangue conforme descrito por Vilches (2009). Imediatamente após serem retirados dos aquários, os peixes foram anestesiados com benzocaína e o sangue foi retirado por um corte na região caudal e, em seguida, foram preparados esfregaços sanguíneos para a confecção de uma lâmina por animal. Após a secagem por 24 horas em temperatura ambiente, as lâminas foram coradas, utilizando-se o corante Panótico Rápido, devido à distinção satisfatória entre eritrócitos, de forma rápida e prática, pois cora o núcleo de azul escuro e o citoplasma de azul claro (Meneguetti et al., 2011). A análise citológica foi realizada usando teste cego (Silva \& Nepomuceno 2010), utilizando-se um estereomicroscópio binocular AXIO/ZEIZZ Model Lab. A1 ${ }^{\circledR}$ com lente de imersão (1000X).

Foram analisados 2000 eritrócitos intactos por lâmina, de acordo com a metodologia proposta por Carrasco et al. (1990) e a determinação da frequência de micronúcleos seguiu os seguintes critérios:

1. tamanho menor que $1 / 3$ do núcleo;

2. mesmo plano de foco do núcleo;

3. padrão da estrutura e coloração da cromatina idênticos ao núcleo;

4. nenhuma ligação com o núcleo.

Durante o experimento, os parâmetros físico-químicos da água nos aquários foram aferidos a cada duas horas (oxigênio dissolvido, $\mathrm{pH}$ e temperatura), com o auxílio de uma sonda multiparâmetro YSI 85. Foram encontrados os valores médios: temperatura $24,5 \pm 0,1^{\circ} \mathrm{C}$; oxigênio dissolvido $4,58 \pm 0,34$ mg L L $;$ pH: 7,8 $\pm 0,1$.

Utilizou-se o teste de Scott-Knott (1974), pelo programa SISVAR (Ferreira, 2011). Sendo considerados significativos os valores de $\mathrm{p}$, ao nível de 5\% entre os tratamentos.

\section{Resultados}

Foi possível observar os micronúcleos (Figura 1) nos eritrócitos de zebrafish em todas as amostras e tempos coletados. 
Nas primeiras seis horas do experimento foi observado que não houve diferença $(\mathrm{P}>0,05)$ para a variação da presença de micronúcleo entre os tratamentos e grupo controle. (Tabela 3). Após 24 horas de experimentação, a frequência média de micronúcleo variou de 11,8 no tratamento controle até o número máximo de 69,2 micronúcleos, no tratamento V. Em 96 horas, a frequência média de micronúcleo foi de 31 no tratamento controle até 147,8 no tratamento VI.

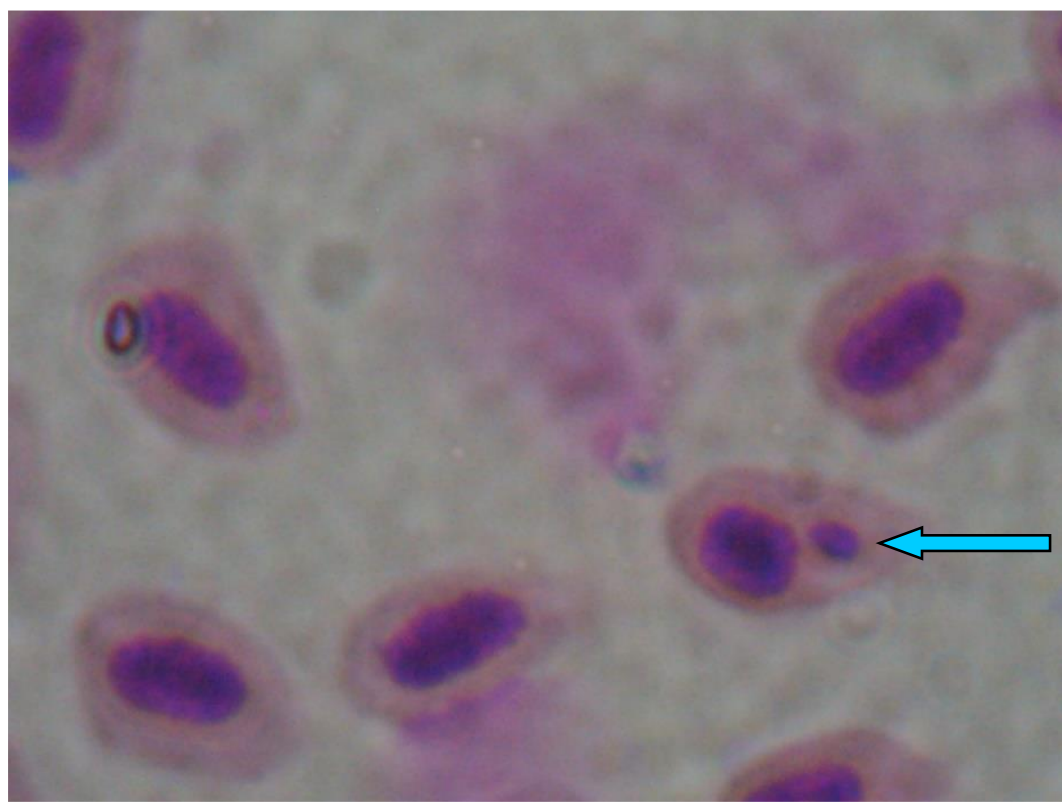

Figura 1. Micronúcleo (seta) em eritrócito de peixe. (100x) (Fote: Arquivo pessoal)

Os dados coletados em 24 horas mostraram que os tratamentos V e VI diferem significativamente (P $>0,05)$ dos demais tratamentos e do grupo controle, o mesmo ocorrendo no tempo de exposição de 96 horas (Tabela 3 ).

Tabela 3. Frequência média de micronúcleos $(n=15)$ em função de 24 e 96 horas de exposição nas diferentes concentrações da FSB.

\begin{tabular}{lcc}
\hline Tratamento & \multicolumn{2}{c}{ Média de micronúcleos* } \\
\cline { 2 - 3 } & 24 horas & 96 horas \\
\hline I (Controle) & $11,8 \mathrm{a}$ & $31,0 \mathrm{a}$ \\
II $\left(0,0645 \mathrm{~g} \mathrm{~L}^{-1}\right)$ & $19,8 \mathrm{a}$ & $60,0 \mathrm{a}$ \\
III $\left(0,129 \mathrm{~g} \mathrm{~L}^{-1}\right)$ & $29,4 \mathrm{a}$ & $34,4 \mathrm{a}$ \\
IV $\left(0,645 \mathrm{~g} \mathrm{~L}^{-1}\right)$ & $32,2 \mathrm{a}$ & $81,4 \mathrm{a}$ \\
V $\left(1,29 \mathrm{~g} \mathrm{~L}^{-1}\right)$ & $69,2 \mathrm{~b}$ & $124,0 \mathrm{~b}$ \\
VI $\left(6,45 \mathrm{~g} \mathrm{~L}^{-1}\right)$ & $65,2 \mathrm{~b}$ & $147,8 \mathrm{~b}$ \\
\hline
\end{tabular}

*Letras minúsculas diferentes na coluna indica diferença significativa a 5\% de probabilidade pelo teste de Scott-Knott.

\section{Discussão}

Considerando que o biodiesel é um combustível que pode vir a substituir o diesel de petróleo, devemse avaliar os riscos da contaminação de corpos hídricos que apontam para o seu potencial tóxico e genotóxico. Pouco se sabe sobre o efeito das frações que compõem o biodiesel sobre os organismos aquáticos, que potencialmente podem entrar em contato por várias rotas de exposição (Abhilash \& Singh, 2009; Birchall et al., 1995; Khan et al., 2007; Peterson \& Möller, 2004; Rekha et al., 2006). Segundo Ramírez \& García( 2005), Campos et al. (2008), Silva \& Nepomuceno (2010), Mendes et al. (2011), Özkan et al. (2011) e Rocco et al. (2012) se a quantidade de micronúcleo for superior a três, a substância é considerada genotóxica. A frequência média de micronúcleo encontrada nesta pesquisa foi de 43,16 no tempo de 24 horas e de 89,52 no tempo de 96 horas, excluindo-se os valores do grupo controle. Portanto, as concentrações da FSB utilizadas podem ser consideradas genotóxicas. 
Conforme Moreira et al. (2010) o estresse causado aos peixes durante a captura, estoque e transporte induz danos genotóxicos e fisiológicos. Sendo este fato atribuído ao mecanismo de formação dos micronúcleos. O que corrobora com os resultados desta pesquisa em que o grupo controle apresentou 11,8 micronúcleos por 2000 células nas primeiras 24 horas e 31 micronúcleos por 2000 células em 96 horas após o início do experimento. Com os resultados observados neste experimento os peixes zebrafish tiveram tolerância a baixas concentrações de FSB, nas primeiras seis horas de experimentação. Alguns autores (Campos et al., 2008; Mendes et al., 2011; Ramírez \& García, 2005) afirmam que o aparecimento de neoplasias está diretamente relacionado com o aumento da frequência de micronúcleos nas células. Mota et al. (2009) e Souza \& Fontanetti (2006) em estudo utilizando tilápias (Oreochromis sp) capturadas diretamente no curso d'água, observaram alta incidência de micronúcleos nos seus eritrócitos. Estas observações foram corroboradas por Silva \& Nepomuceno (2010) que, pesquisando mandis amarelo (Pimelodus maculatus), encontraram um número de micronúcleos compreendido entre 56 e 226, evidenciando que a formação do micronúcleo pode estar relacionada com a contaminação da água dos rios.

No presente estudo foi observado que nos tempos de 24 e 96 horas de exposição, houve diferença (P $<0,05)$ para a variação da presença de micronúcleo nos tratamentos V e VI em relação aos outros tratamentos e o tratamento controle. Esses resultados são semelhantes aos relatados por Özkan et al. (2011) e Rocco et al. (2012) que encontraram um aumento do dano genético em relação ao tempo de exposição, ou seja, quanto maior for o tempo de exposição, maior é o dano. Mesmo pesquisando o efeito de substâncias diferentes.

Em pesquisa realizada por Khan et al. (2007), sobre os efeitos toxicológicos causados pela fração solúvel em água (FSA) do biodiesel (derivado de óleos e gorduras recicláveis), óleo diesel e de misturas de ambos sobre organismos testes Oncorhynchus mykiss (peixe truta arco-íris) e de dáfinia (Daphnia magna), através da realização de testes de toxicidade aguda, comprovaram que a FSA do biodiesel é consideravelmente menos tóxica para os organismos aquáticos do que a FSA do óleo diesel convencional. Porém, os resultados demonstraram que ainda assim há risco substancial para os organismos aquáticos, no caso de uma contaminação por biodiesel.

\section{Conclusão}

A fração solúvel do biodiesel de óleo de soja queimado tem efeito genotóxico nos eritrócitos de juvenis de zebrafish com maior frequência de micronúcleos a partir da concentração de $1,29 \mathrm{~g} \mathrm{~L}-{ }^{1}$, em 24 e 96 horas de exposição.

\section{Referências bibliográficas}

Abhilash, P. C., \& Singh, N. (2009). Pesticide use and application: an Indian scenario. Journal of Hazardous Materials, 165(1-3), 1-12.

Al-Sabti, K., \& Metcalfe, C. D. (1995). Fish micronuclei for assessing genotoxicity in water. Mutation Research/Genetic Toxicology, 343(2-3), 121-135.

Birchall, C., Newman, J. R., \& Greaves, M. P. (1995). Degradation and phytotoxicity of biodiesel oil. Institute of Arable Crops Research. Report, Long Ashton Research Station, Bristol, UK, 1, 50.

Campos, L. M. F. R., Dias, F. da L., Antunes, L. M. G., \& Murta, E. F. C. (2008). Prevalence of micronuclei in exfoliated uterine cervical cells from patients with risk factors for cervical cancer. Sao Paulo Medical Journal, 126(6), 323-328.

Carrasco, K. R., Tilbury, K. L., \& Myers, M. S. (1990). Assessment of the piscine micronucleus test as an in situ biological indicator of chemical contaminant effects. Canadian Journal of Fisheries and Aquatic Sciences, 47(11), 2123-2136.

Fenech, M. (1997). The advantages and disadvantages of the cytokinesis-block micronucleus method. Mutation Research/Genetic Toxicology and Environmental Mutagenesis, 392(1-2), 11-18.

Ferreira, D. F. (2011). SISVAR: A Computer Statistical Analysis System. Ciência e Agrotecnologia, 35(6), 1039-1042. https://doi.org/http://dx.doi.org/10.1590/S1413-70542011000600001.

Khan, N., Warith, M. A., \& Luk, G. (2007). A comparison of acute toxicity of biodiesel, biodiesel 
blends, and diesel on aquatic organisms. Journal of the Air \& Waste Management Association, 57(3), 286-296.

Masouleh, F. F., Amiri, B. M., Mirvaghefi, A. R., \& Nemtollahi, M. A. (2011). In vitro effects of diazinon on male reproductive tissue and sperm motility of Caspian kutum (Rutilus frisii kutum). Research Journal of Environmental Toxicology, 5(2), 108-116.

Mendes, C. F., Gardinalli Filho, G., Furoni, R. M., Miranda, L. V. V., Boschini Filho, J., \& Sampaio Neto, L. F. (2011). Micronúcleos em células do colo uterino em mulheres HIV+ segundo sua condição de imunidade. Revista Brasileira de Ginecologia e Obstetrícia, 33(10), 305-309.

Meneguetti, D. U. O., Silva, F. C., Zan, R. A., Poletto, P. O., \& Ramos, L. J. (2011). Adaptação da técnica de micronúcleo em allium cepa, para futuras análises de mutagenicidade dos rios da região do Vale do Jamari, Rondônia, Amazônia Ocidental. Revista Pesquisa \& Criação, 10(2), 181-187.

Moreira, T. N., Nunes, E. A., Leal, M. E., Schulz, U. H., \& Lemos, C. T. (2010). Influência dos métodos de captura de peixes na avaliação genotóxica utilizando diferentes tecidos de Astyanax fasciatus (Osteichthyes, Characidae). Journal of Brazilian Society of Ecotoxicology, 5, 1-7.

Mota, G. G. P., Barboni, S. A. V., \& Jesús, M. C. (2009). Tilápias (Actinopterygii: Cichlidae) comercializadas em feira de Santana (Bahia) como bioindicadores de poluição ambiental em rios da bacia do Paraguaçu. Pesticidas: Revista de Ecotoxicologia e Meio Ambiente, 19.

Özkan, F., Gündüz, S. G., Berköz, M., \& Özlüer, H. A. (2011). Induction of micronuclei and other nuclear abnormalities in peripheral erythrocytes of Nile tilapia, Oreochromis niloticus, following exposure to sublethal cadmium doses. Turkish Journal of Zoology, 35(4), 585-592.

Peterson, C. L., \& Möller, G. (2004). Biodegradability, BOD5, COD and toxicity of biodiesel fuels. University of Idaho Biodiesel Education.

Ramírez, O. A. B., \& García, F. P. (2005). Genotoxic damage in zebra fish (Danio rerio) by arsenic in waters from Zimapan, Hidalgo, Mexico. Mutagenesis, 20(4), 291-295.

Rekha, Naik, S. N., \& Prasad, R. (2006). Pesticide residue in organic and conventional food-risk analysis. Journal of Chemical Health \& Safety, 13(6), 12-19.

Rocco, L., Peluso, C., \& Stingo, V. (2012). Micronucleus test and comet assay for the evaluation of zebrafish genomic damage induced by erythromycin and lincomycin. Environmental Toxicology, 27(10), 598-604.

Santos, F. A. (2010). Biocombustíveis: prós e contras. UTAD Publicações.

Silva, A. C., \& Nepomuceno, J. C. (2010). Avaliação da frequência de micronúcleos em eritrócitos periféricos de mandi-amarelo (Pimelodus maculatus) do rio Paranaíba. Perquirere (UNIPAM), 1 , $167-179$.

Simonato, J. D., Albinati, A. C., \& Martinez, C. B. R. (2006). Effects of the water soluble fraction of diesel fuel oil on some functional parameters of the neotropical freshwater fish Prochilodus lineatus Valenciennes. Bulletin of Environmental Contamination and Toxicology, 76(3), 505-511.

Souza, T. S., \& Fontanetti, C. S. (2006). Micronucleus test and observation of nuclear alterations in erythrocytes of Nile tilapia exposed to waters affected by refinery effluent. Mutation Research/Genetic Toxicology and Environmental Mutagenesis, 605(1-2), 87-93.

Swanson, K. J., Madden, M. C., \& Ghio, A. J. (2007). Biodiesel exhaust: the need for health effects research. Environmental Health Perspectives, 115(4), 496-499.

Vilches, M. (2009). Análise genotóxica do Rio Cadeia/RS através do ensaio cometa e teste de micronúcleo e anormalidades nucleares utilizando peixes como bioindicadores. Centro Universitário de Feevale.

Recebido: 12 de dezembro, 2019

Aprovado: 26 de janeiro, 2020 .

Publicado: 15 maio 2020 .

Licenciamento: Este artigo é publicado na modalidade Acesso Aberto sob a licença Creative Commons Atribuição 4.0 (CC-BY 4.0), a qual permite uso irrestrito, distribuição, reprodução em qualquer meio, desde que o autor e a fonte sejam devidamente creditados. 